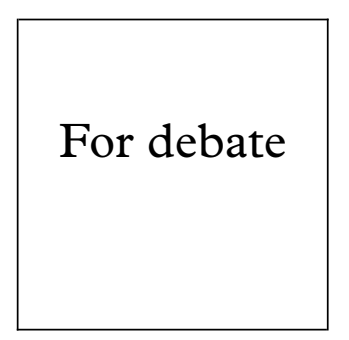

\author{
Noreen M Desmond, Richard J Coker
}

\author{
The case for
}

\title{
Should preventive antiretroviral treatment be offered following sexual exposure to HIV?
}

\begin{abstract}
Introduction
Although not conclusive, the data supporting the use of antiretroviral therapy following occupational exposure to HIV in order to reduce the risk of seroconversion are compelling. ${ }^{1}$ As a consequence American and, more recently, United Kingdom guidelines on the use of post-exposure prophylaxis (PEP) by healthcare workers have been produced. ${ }^{2}{ }^{3}$ There are, however, no guidelines for nonoccupational exposure, such as sexual exposure. The risk of acquiring HIV infection from a single episode of unprotected receptive anal or vaginal intercourse with an infected individual is similar to that following a needlestick injury. ${ }^{4}$ The most common route of HIV infection is sexual and increasingly, in practice, clinicians are faced with decisions regarding the provision of prophylactic treatment after possible or probable sexual exposure. ${ }^{5}$
\end{abstract}

\section{What is the efficacy of PEP in cases of sexual exposure to HIV infection?}

Although there are few data, similar immunological responses probably occur whether exposure is mucosal or transcutaneous. ${ }^{6}$ It seems likely that antiretroviral therapy would, therefore, reduce the risks of HIV infection following sexual exposure from approximately 1 in 300. A case-control study suggested that zidovudine alone reduced the risk of HIV infection by $79 \%$ in occupational exposures. ${ }^{1}$ Combinations of reverse transcriptase inhibitors with the addition of a protease inhibitor are expected to reduce the risks further and, similarly, would be expected to reduce the risks for those exposed sexually. However, there have been no formal studies of any kind to assess the efficacy of prophylactic antiretroviral therapy in sexual settings, although researchers in San Francisco are awaiting ethical approval and funding to set up a post-exposure treatment programme. ${ }^{7}$ The study will be too small to show whether the preventive treatment works, but should shed light on how often people take such risks, how quickly they will come forward, and what proportion choose to take PEP. Furthermore, given our knowledge to date, it is probably unethical to withhold treatment following sexual exposure but give it following occupational exposure.

\section{What policy should be adopted?}

We believe that "emergency prophylaxis" should be offered in cases of high risk sexual exposure, which we would define as unpro- tected anal or vaginal intercourse to ejaculation with an HIV infected partner. The antiretroviral regimen should be the same as recommended for occupational exposure to HIV infection $^{3}$ and obviously only given with the patient's informed consent.

What about after rape $e^{8}$ or in cases of high probability but where there is uncertainty over HIV status?

We would, in addition, propose that PEP is offered to victims of sexual assault or rape if the assailant is known or strongly suspected of being HIV seropositive. Similar recommendations have been formulated in the United States. ${ }^{9}$ If the sexual contact or the patient's HIV status is unknown, initiating PEP should be decided on a case by case basis, depending upon on the type of exposure and the likelihood of HIV transmission in the sexual contact.

\section{What are the practical difficulties of implementing PEP?}

Efficacy of prophylaxis is probably maximised if therapy is initiated promptly. ${ }^{10}$ The key to this issue is immediate 24 hour access to chemoprophylaxis. ${ }^{5}$ Genitourinary medicine services are ideally placed, being open access, to provide post-sexual exposure prophylaxis and to coordinate, according to local expertise and logistics, "out of hours" care probably through their accident and emergency department. Public health campaigns would be required to inform individuals about the need to contact appropriate medical services promptly. This could be implemented as part of the overall programme of health promotion and HIV prevention.

\section{Should serodiscordant couples have emergency prophylaxis packs at home in case of condom failure?}

We would urge that preventative treatment should be used judiciously and thus provision of home treatment is probably inappropriate. With antiretroviral treatment becoming increasingly complex consultation with an expert physician is advisable with each episode of potential transmission.

\section{What are the risks of PEP?}

Although serious toxicity is uncommon with zidovudine alone, combination treatment with newer drugs increases the frequency of side effects and potential drug interactions. Of note 
in one study, one in three healthcare workers stopped therapy because of undesirable side effects. ${ }^{11}$ Furthermore, the long term consequences of taking multiple antiretroviral drugs are unknown. The choice of treatment will need to take account of the sexual partner's antiretroviral drug history because of the possibility of transmission of virus already resistant to some drugs. In addition, consideration should be given to the possibility that antiretroviral resistance will be encouraged with the wider use of drugs.

Would unsafe sex increase as individuals are lulled into a false sense of security that there is an effective "morning after" treatment for HIV? Similar fears were expressed regarding emergency contraception but there is no evidence that behaviour changed (Anna Glasier, personal communication). One might equally argue that individuals who have undergone the anxiety of possible infection with HIV and the inconvenience and toxicities of a complex treatment regimen may be less likely to place themselves at risk in the future.

\section{What are the costs of PEP?}

There are significant cost implications in adopting a policy of post-sexual exposure prophylaxis although these may, to some extent, be offset by reduced transmission rates. A month's supply of triple combination therapy costs approximately $£ 700$. However, since it may be necessary to provide chemoprophylaxis for about 300 exposures in order to prevent one potential seroconversion, the true cost per infection prevented may be closer to $£ 200000$. Treatment may well be cheaper than chemoprophylaxis.

Should scarce resources be expended when the value of the intervention is still uncertain?

The intervention would be made more cost effective by targeting PEP towards individuals with the highest risk exposures as we have recommended and using shorter courses of treatment which may be equally effective in prophylaxis. This dilemma also applies to occupational exposures.

Who will pay for PEP?

Hospitals have had to address the resource implications for PEP for occupational exposures and this could be expanded locally to include post-sexual exposure prophylaxis. However, convincing purchasers that this is a priority will no doubt be difficult especially as they are keen only to fund evidence based medicine. An alternative, which we would favour, would be national guidelines supported by all purchasers and trusts.

\section{Conclusion}

Since current recommendations on chemoprophylaxis have side stepped the issue of nonoccupational exposure there remains a need for national guidelines to support professional practice. Such guidelines could be implemented and monitored through a national or international body, so that management is coordinated and not haphazard as it is at present, and failures of prophylaxis noted early. It also seems appropriate to create a register for non-occupational exposures to HIV infection similar to that for occupational exposure. Finally, there is a need to educate both healthcare professionals and the public about when and where to go for appropriate post-exposure management.

1 Centers for Disease Control. Case-control study of HIV seroconversion in healthcare workers after percutaneous exposure to HIV-infected blood-France, United Kingdom and United States, January 1988-August 1994. MMWR 1995;44:929-33.

2 Centers for Disease Control. Update: Provisional Public Health Service recommendations for chemoprophylaxis after occupational exposure to HIV. MMWR 1996;45:46872 .

3 Department of Health. Guidelines on post-exposure prophylaxis for health care workers occupationally exposed to HIV. London: DoH, June 1997.

$4 \mathrm{Katz} \mathrm{MH}$, Gerberding JL. Post exposure treatment of people exposed to the human immunodeficiency virus through sexual contact or injection-drug use. $N$ Engl f Med 1997;336:1097-100.

5 Desmond NM, King ECJ, Dawson SG. Sexual exposure to HIV infection: is there a role for emergency prophylaxis? Int f STD AIDS 1998;9:51-2.

6 Blauvelt A. A role of skin dendritic cells in the initiation of human immunodeficiency virus infection. Am f Med 1997; 102:16-20.

7 Macready N. Morning-after AIDS treatment. Lancet 1997;349:78.

8 Murphy S, Kitchen K, Harris JRW, et al. Rape and subsequent seroconversion to HIV. BMF 1989;299:718.

9 Gostin LO, Lazzarini Z, Alexander D, et al. HIV testing, Gostin LO, Lazzarini Z, Alexander $\mathrm{D}$, et al. HIV testing,
counselling and prophylaxis after sexual assault. $\mathscr{f} A M A$ counselling and propt

10 Martin LN, Murphy-Corb M, Soike KF, et al. Effects of initiation of 3'-azido, 3'-deoxythymidine (zidovudine) treatment at different times after infection of rheusus monkeys with simian immunodeficiency virus. F Infect Dis 1993;168 825-35.

11 Tokars JI, Marcus R, Culver DH, et al. Surveillance of HIV infection and zidovudine use among health care workers after occupational exposure to HIV infected blood. Ann Intern Med 1993;118:913-9. 